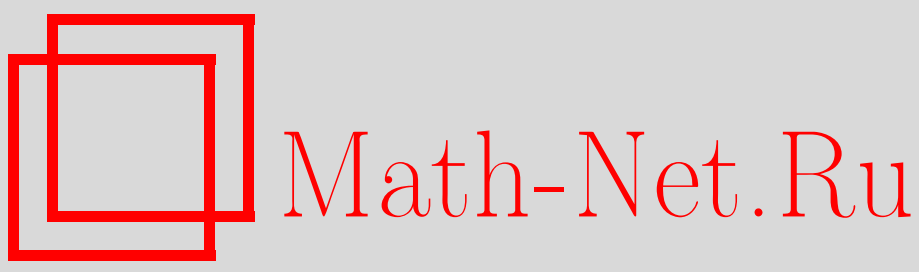

A. I. Sozutov, Two observations on groups with engel elements, Sibirsk. Mat. Zh., 2019, Volume 60, Number 6, 1411-1413

DOI: https://doi.org/10.33048/smzh.2019.60.617

Use of the all-Russian mathematical portal Math-Net.Ru implies that you have read and agreed to these terms of use http://www . mathnet.ru/eng/agreement

Download details:

IP : 3.91 .87 .62

April 26, 2023, 17:47:17

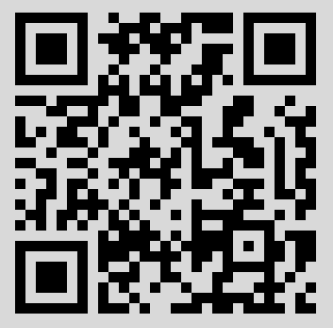


Сибирский математический журнал

Ноябрь-декабрь, 2019. Том 60, № 6

УДК 512.542

\title{
ДВА ЗАМЕЧАНИЯ О ГРУППАХ С ЭНГЕЛЕВЫМИ ЭЛЕМЕНТАМИ
}

\section{А. И. Созутов}

\begin{abstract}
Аннотация. Даны ответы (отрицательные) на вопросы 16.15a) и 17.3 из «Коуровской тетради»: «во всякой ли группе множество всех ограниченно энгелевых элементов является подгруппой?» и «верно ли, что группа бинарно нильпотентна, если в ней любое 4-элементное подмножество содержит два элемента, порождающие нильпотентную подгруппу?».
\end{abstract}

DOI 10.33048/smzh.2019.60.617

Ключевые слова: энгелевы группы, энгелевы и ограниченно энгелевы элементы.

Элемент $a$ произвольной группы $G$ называется энгелевым, если для любого элемента $b \in G$ существует такое зависящее от него натуральное число $n=n(b)$, что выполняется равенство $[\ldots[[b, a], a], \ldots, a]=\left[b,{ }_{n} a\right]=1$; если при этом число $n$ можно выбрать одно и то же для всех $b \in G$, то элемент $a$ называется ограниченным энгелевым или $n$-энгелевым. Группа, состоящая из энгелевых (n-энгелевых) элементов, называется энгелевой (n-энгелевой) [1, Д26]. Свойства энгелевых элементов и их расположение в группе изучались многими авторами. Составляют ли энгелевы элементы подгруппу (радикал $[1$, Д20]) - один из основных рассматриваемых вопросов. Исследования групп с энгелевыми элементами стимулируются также изучением групп с различными условиями конечности и групп с элементами малых порядков (см., например, $[1$, Д26] и комментарии к вопросам 9.50, 11.11 из «Коуровской тетради» [2]).

Группа $G$ с энгелевым элементом $a$ порядка 2 удовлетворяет условиям теоремы Бэра - Сузуки, поскольку подгруппы вида $\left\langle a, a^{g}\right\rangle$ в $G$ являются конечными 2-подгруппами. Действительно, $[b, a] \in\left\langle a, a^{b}\right\rangle$ и $\left[b,{ }_{n} a\right] \in\left\langle a, a^{b}\right\rangle$ для всех $n$, и равенства $|a|=2$ и $\left[b,{ }_{n} a\right]=1$ возможны только в случае, когда порядок группы диэдра $\left\langle a, a^{b}\right\rangle$ равен $2^{m}$, где $m \leq n$. Поэтому в любой 2-группе инволюции являются энгелевыми элементами, а в группе периода $2^{n}-(n+1)$-энгелевыми. Для любого $n \geq 100$ существуют простые 2-группы периода $2^{n}$ (см. [2, архив, 4.74a]), порожденные $(n+1)$-энгелевыми элементами.

С использованием конструкции $n$-периодического произведения для достаточно большого четного $n$ [3] в [4] построены бесконечные простые непримарные группы ограниченного четного периода, в которых произведение любых двух инволюций есть 2-элемент. В этих группах каждая инволюция является конечным ограниченным энгелевым элементом. Группы из $\S 3$ работы [4] (контрпримеры к вопросу 11.11(a) А. В. Боровика из [2]) также дают отрицательный ответ на следующий вопрос В. В. Блудова 16.15a) из [2].

Исследование выполнено при финансовой поддержке РФФИ в рамках научного проекта № 19-01-00566 A.

(c) 2019 Созутов А. И. 
16.15. Элемент $g$ группы $G$ называется энгелевым, если для каждого элемента $h \in G$ существует такое $k$, что $[h, g, \ldots, g]=1$, где $g$ встречается $k$ раз; если существует $k$, не зависящее от $h$, то $g$ называется ограниченно энгелевым.

(a) (Б. И. Плоткин). Во всякой ли группе множество всех ограниченно энгелевых элементов является подгруппой?

В качестве контрпримера $G$ к вопросу вначале возьмем удобный для доказательства пример - подгруппу $N$ из теоремы $3(2)$ в [4]. Согласно теореме группа $G=N$ порождена элементами порядка 3 и проста и каждая конечная подгруппа в $G$ либо удовлетворяет тождеству $x^{2^{48}}=1$, либо имеет порядок 3 . Значит, инволюции в $G$ являются ограниченно энгелевыми элементами и в силу простоты группа $G$ ими порождена.

Пусть $b$ - элемент порядка 3 из $G$ и $a \in G \backslash\langle b\rangle$. Допустим, что $\left[a,{ }_{k} b\right]=1$, где $k$ - минимальное с данным свойством число, и обозначим $g=\left[a,{ }_{k-2} b\right]$. В силу свойств группы $G$ имеем $\left[a,{ }_{k-1} b\right] \in\langle b\rangle,\left[a,{ }_{k-1} b\right]=g^{-1} b^{-1} g b=b^{\alpha}, \alpha= \pm 1$ и $g^{-1} b^{-1} g=b^{\alpha-1}$, т. е. $g \in N_{G}(\langle b\rangle)$. Но тогда $g \in\langle b\rangle$ в силу свойств группы $G$ и, значит, $\left[a,{ }_{k-1} b\right]=1$ вопреки выбору числа $k$. Следовательно, элемент $b$ не является энгелевым, и в группе $G$ множество всех ограниченных энгелевых элементов не является подгруппой.

Контрпримерами к вопросу 16.15(а) также являются некоторые из $n$-периодических произведений $G=\prod_{l \in I}^{n} G_{l}$ теоремы 2 из [4], в которых $n=2^{m} \geq 2^{48}$, $|I| \geq 2$, а сомножители $G_{l}$ - нетривиальные периодические группы без инволюций. По $[4$, лемма 1$]$ любая конечная подгруппа из $G$ сопряжена с подгруппой из некоторого множителя $G_{l}$ или в ней выполняется тождество $x^{n}=1$. Если, например, один из множителей $G_{l}$ является циклической $p$-группой $\langle b\rangle$, а другие множители $G_{l}$ не содержат $p$-элементов, то в силу доказанного выше элемент $b$ не энгелев в $G$.

Наконец, воспользуемся тем, что каждый из множителей $n$-периодического произведения $G=\prod_{l \in I}^{n} G_{l}$ канонически вложен в $G$ [4, теорема Сонкина]. Если неединичный элемент $b \in G_{l}$ не является энгелевым в множителе $G_{l}$, то он не будет энгелевым и в $G$. Отрицательный ответ на вопрос $16.15(\mathrm{a})$, видимо, можно получить также в классе 2-групп, но автор не знает такого доказательства.

В 17-м издании «Коуровской тетради» [2] А. Абдоллахи поставил следующий вопрос.

17.3. Пусть $G$ - группа, в которой любое 4-элементное подмножество содержит два элемента, порождающие нильпотентную подгруппу. Верно ли, что любая 2-порожденная подгруппа группы $G$ нильпотентна?

Пусть $x, y$ - произвольные элементы группы $G$, удовлетворяющей условиям вопроса, и допустим, что подгруппа $\langle x, y\rangle$ не нильпотентна. Тогда $y \neq x^{-1} y x$, и так как

$$
\langle x, y\rangle=\langle x, y x\rangle=\langle y, y x\rangle=\left\langle x, x^{-1} y x\right\rangle=\left\langle y x, x^{-1} y x\right\rangle,
$$

подмножество $\left\{x, y, y x, x^{-1} y x\right\}$ состоит из четырех различных элементов и в силу условий подгруппа $\left\langle y, x^{-1} y x\right\rangle$ нильпотентна. Из этого следует, что в $G$ любые два сопряженных элемента порождают нильпотентную подгруппу и $G-$ энгелева группа.

Далее, пусть при тех же предположениях, что и выше, $x^{2} \neq 1$. Тогда

$$
\langle x, y\rangle=\langle x, y x\rangle=\langle y, y x\rangle=\left\langle x, y x^{2}\right\rangle=\left\langle y x, y x^{2}\right\rangle,
$$


элементы $x, y, y x, y x^{2}$ различны и подгруппа $\left\langle y, y x^{2}\right\rangle=\left\langle y, x^{2}\right\rangle$ нильпотентна. Отсюда следует, что каждый элемент конечного нечетного порядка с любым элементом (произвольного порядка) порождает в $G$ нильпотентную подгруппу. В частности, для периодических групп без инволюций вопрос 17.3 имеет положительное решение.

Укажем конкретный контрпример к вопросу 17.3 в классе 2-групп. В [5, предложение 1] среди других примеров построены 2-порожденные бесконечномерные финитно-аппроксимируемые ассоциативные нильалгебры вида $A=$ $\left\langle\left\langle x, y \mid f_{1}, f_{2}, \ldots\right\rangle\right\rangle$ над полем $\Phi$ из двух элементов, в которых каждая подалгебра, порожденная тремя элементами из $A^{2}$, нильпотентна. Пусть $G=\langle x, y\rangle-$ подгруппа присоединенной группы алгебры $A$ и $H=G \cap A^{2}$. Тогда $G$ - бесконечная финитно-аппроксимируемая 2-группа, $H$ - нормальная в $G$ подгруппа индекса 4 и фактор-группа $G / H$ элементарная абелева.

Покажем, что группа $G$ удовлетворяет условиям вопроса 17.3 . В силу теорем Пуанкаре и Бернсайда о базисе [6, теорема 12.2.1] каждая максимальная подгруппа $M$ в $G$, имеющая конечный индекс, нормальна в $G$ и содержит подгруппу $H$. Поэтому в $G$ точно три максимальные нормальные подгруппы индекса 2, это $X=\langle x, H\rangle, Y=\langle y, H\rangle$ и $Z=\langle x y, H\rangle$ (здесь через $x y$ обозначено групповое произведение относительно операции присоединенного умножения). В силу свойств алгебры $A^{2}$ в подгруппе $H$ любые три элемента порождают конечную 2-подгруппу. Если $f \in G \backslash H$ и $g \in H$, то $f^{2} \in H$ и подгруппа $K=\left\langle f^{2}, g, f^{-1} g f\right\rangle$ конечна и нормальна в подгруппе $L=\langle f, g\rangle$. Поскольку $[L: K]=2$, подгруппа $L=\langle f, g\rangle$ конечна. Отсюда следует, что подгруппы $X$, $Y$ и $Z$ бинарно конечны (2-нильпотентны). Учитывая равенство $G=X \cup Y \cup Z$, приходим к выводу, что любое 4-элементное подмножество из $G$ содержит не менее двух общих элементов хотя бы с одной из подгрупп $X, Y, Z$. В силу доказанного выше эти два общих элемента порождают нильпотентную подгруппу. Таким образом, группа $G$ удовлетворяет условиям вопроса 17.3 А. Абдоллахи и является к нему контрпримером.

\section{ЛИТЕРАТУРА}

1. Курош А. Г. Теория групп. М.: Наука, 1967.

2. Коуровская тетрадь. Нерешенные вопросы теории групп. 19-е изд. Новосибирск: Ин-т математики СО РАН, 2018.

3. Sonkin D. M. On groups of large exponents $n$ and $n$-periodic products: $\mathrm{PhD}$ Thez, Vanderbild Univ., Nashville, Tennessee, 2005, 83 pp. etd.library.vanderbild/edu/avialable/etd-06032005025224/.

4. Мазуров В. Д., Ольшанский А. Ю., Созутов А. И. О бесконечных группах конечного периода // Алгебра и логика. 2015. Т. 54, № 2. С. 243-251.

5. Созутов А. И. О примерах ассоциативных нильалгебр // Мат. заметки. 1995. Т. 57, № 3. C. $445-450$.

6. Холл М. Теория групп. М.: Изд-во иностр. лит., 1962.

Поступила в редакцию 7 декабря 2018 г.

После доработки 6 марта 2019 г.

Принята к публикации 12 марта 2019 г.

Созутов Анатолий Ильич

Сибирский федеральный университет, пр. Свободный, 79, Красноярск 660041

sozutov_ai@mail.ru 